\title{
Protective effect of naringin against ankylosing spondylitis via ossification, inflammation and oxidative stress in mice
}

\author{
KANG LIU, LIANGUO WU, XIAOLIN SHI and FENGQING WU \\ Department of Orthopedics, The Second Hospital Affiliated to Zhejiang University of Chinese Medicine, \\ Hangzhou, Zhejiang 310005, P.R. China
}

Received December 16, 2014; Accepted February 4, 2016

DOI: $10.3892 /$ etm.2016.3410

\begin{abstract}
Naringin is an abundant flavanone in pomelo, grapefruit as well as lime and its variants, has been shown to exhibit certain antioxidative, anti-inflammatory, anti-cancer and hypoglycemic effects. The aim of the current study was to evaluate the protective effects of naringin against ankylosing spondylitis (AS) and to elucidate the potential underlying mechanism. Firstly, a mouse model of ankylosing spondylitis (AS) was established. Next, osteocalcin (OC), alkaline phosphatase (ALP) and triglyceride (TG) activity values, inflammatory factor and oxidative stress were evaluated in the AS mice. Then, the Janus kinase 2 (JAK2) and signal transducer and activator of transcription 3 (STAT3) protein expression levels in the AS mice were investigated using western blot analysis. The results showed that naringin increased OC, ALP and TG activity values in the AS mouse model. Furthermore, inflammatory factor and oxidative stress levels in the AS mice were restrained by treatment with naringin. Furthermore, JAK2 and STAT3 protein expression levels were reduced by treatment with naringin. In conclusion, the present results indicated that the protective effects of naringin against AS are exerted via the induction of ossification, suppression of inflammation and oxidative stress and the downregulation of JAK2/STAT3 in mice.
\end{abstract}

\section{Introduction}

Ankylosing spondylitis (AS) is an autoimmune disease characterized by sacroiliitis and spinal rigidity, which attack the axial joints (1). Clinical symptoms include the pain in areas including the lumbar, back, shoulder and neck, with or

Correspondence to: Mr. Lianguo Wu, Department of Orthopedics, The Second Hospital Affiliated to Zhejiang University of Chinese Medicine, 318 Chaowang Road, Hangzhou, Zhejiang 310005, P.R. China

E-mail: lianguowud1@163.com

Key words: naringin, ankylosing spondylitis, ossification, inflammation, oxidative stress without spasticity. Furthermore, these can include heel pain, pain aggravation at night, even nocturnal awakening, turning difficult and obvious spasticity on waist in the early morning or after sedentary state (2). AS is a systemic autoimmune disease, with final outcomes including ossification of the ligaments around the spine and intervertebral disk, and the fibrosis of axial joints and bony ankylosis, leading to the disability of joint movement (3). Therefore, as pathological osteogenesis is the predominant cause of disability in AS patients, the treatment of AS may need to address the rigidity problems in normal ligament and synovial ossification (4).

Thrombocytosis may also occur in AS patients, which is known as reactive thrombocytosis (5). Thrombocytosis is also an inflammatory process, the interactions of various cytokines result in megakaryocytic hyperplasia, leading to an increase in platelet count and platelet-large cell ratio (6). However, in a clinical context, the inhibition of platelets in patients with AS is lower compared with that of rheumatoid arthritis (7).

Reactive oxygen species and reactive nitrogen species are harmful free radicals generated by metabolic processes, which can induce oxidative stress (8). Superoxide dismutase (SOD), catalase (CAT), and glutathione peroxidase (GSH-PX) are key antioxidant enzymes; SOD can remove superoxide radicals from biological cells by disproportionation, generating $\mathrm{H}_{2} \mathrm{O}_{2}$ and $\mathrm{O}_{2}$, and $\mathrm{H}_{2} \mathrm{O}_{2}$ may be catalyzed by CAT to generate $\mathrm{H}_{2} \mathrm{O}$ and $\mathrm{O}_{2}$, thereby reducing the toxicity of free radicals on the organism (9).

Naringin is (full name, naringenin-7-O-neohesperidin glycoside) is a dihydrogen flavonoid, and the primary active ingredient of a number of traditional Chinese medicines, including orange, Drynaria and Citrus aurantium. Naringin exhibits a wide range of biological activities, including anti-oxidation (10), bone growth promotion (11), plasma cholesterol reduction (12), anti-atherosclerotic (13), sedative (14), anti-tumor (15) and anti-fungal (16) properties. However, the protective effect of naringin against AS and the underlying molecular mechanism, including the signal pathways affecting ossification, inflammation and oxidative stress, remain unclear. The present study aimed to evaluate the protective effect of naringin against ossification, inflammation and oxidative stress, and to further investigate the possible underlying mechanisms, including anti-inflammatory signaling pathways. 


\section{Materials and methods}

Materials and chemicals. Osteocalcin (OC; ml026391), alkaline phosphatase (ALP; E-CL-M0075c) and triglyceride (TG; E-EL-M2603c), nuclear factor (NF)- $\kappa B$ p65 unit (ml026326), tumor necrosis factor- $\alpha$ (TNF- $\alpha$; m1022566), interleukin- $1 \beta$ (IL-1 $\beta$; ml028611), IL-6 (ml028608), malonaldehyde (MDA; ml027131), SOD (m1026976), CAT (E-EL-H5408c) and GSH-PX (E-EL-H5410c) enzyme-linked immunosorbent assay (ELISA) determination kits were obtained from the Beyotime Institute of Biotechnology (Nanjing, China). A bicinchoninic acid (BCA; 5000001) assay kit was obtained from Bio-Rad Laboratories, Inc. (Hercules, CA, USA). Naringin (purity, $>95 \%$ ) was purchased from Sigma-Aldrich (Sigma-Aldrich). The chemical structure of naringin is shown in Fig. 1.

Construction of AS mouse model. All animal studies were performed in accordance with the regulations of the Zhejiang University Hospital for the care and use of laboratory animals (Shandong, China). Kunming mice were obtained from the Laboratory Animal Institute of The Second Hospital Affiliated to Zhejiang University of Chinese Medicine (Hangzhou, China). The AS model was established in the mice as described previously (17). A total of 50 mice were injected with 30 UI human chorionic gonadotropin hormone (HCG; Sigma-Aldrich) to induce superovulation. Then, zygotes were gathered and a HLAB2704 gene fragment was injected using microinjection into the pronucleus. Surviving zygotes were transferred into pseudocyesis mice for generation. In brief, AS model mice were prepared by Biocytogen Biological Technology Co. Ltd. (eijing, China). A total of 50 mice were injected with HCG hormone to induce superovulation. Next, ovum and sperm were integrated, zygotes were gathered and HLAB2704 gene fragments were injected using a microinjection into the pronucleus. Surviving zygotes were transferred into pseudocyesis mice for generation. The mice were sacrificed by incision under $50 \mathrm{mg} / \mathrm{kg}$ of sodium pentobarbital.

Grouping and treatment. Five treatment groups were established for the study ( $\mathrm{n}=10$ per group), as follows: i) Control group, which included normal mice that received $0.1 \mathrm{ml} / 100 \mathrm{~g}$ sodium pentobarbital (Sigma-Aldrich) injected intraperitoneally (i.p.); ii) AS group, which included AS mice that received $0.1 \mathrm{ml} / 100 \mathrm{~g}$ sodium pentobarbital (i.p.); iii) Nar (20) group, which included AS mice that received $20 \mathrm{mg} / \mathrm{kg}$ naringin (i.p.) for 8 days (18); iv) Nar (40) group, which included AS mice that received $40 \mathrm{mg} / \mathrm{kg}$ naringin (i.p.) for 8 days; and v) Nar (80), which included AS mice that received $80 \mathrm{mg} / \mathrm{kg}$ naringin (i.p.) for 8 days.

ELISA of OC, ALP and TG activity. Following treatment with naringin for 3 days, OC, ALP and TG, NF- $\mathrm{B}$ p 65 unit, TNF- $\alpha$, IL- $1 \beta$ and IL-6, (inflammatory factors); MDA, SOD, CAT and activity of GSH-PX (oxidative stress) activity were determined using ELISA determination kits, according to the standard curve (Beyotime Institute of Biotechnology, China (Liu, Fan). The optical density was read at $405 \mathrm{~nm}$ using a Bio-Rad microplate reader (Bio-Rad Laboratories, Inc., Hercules, CA, USA).

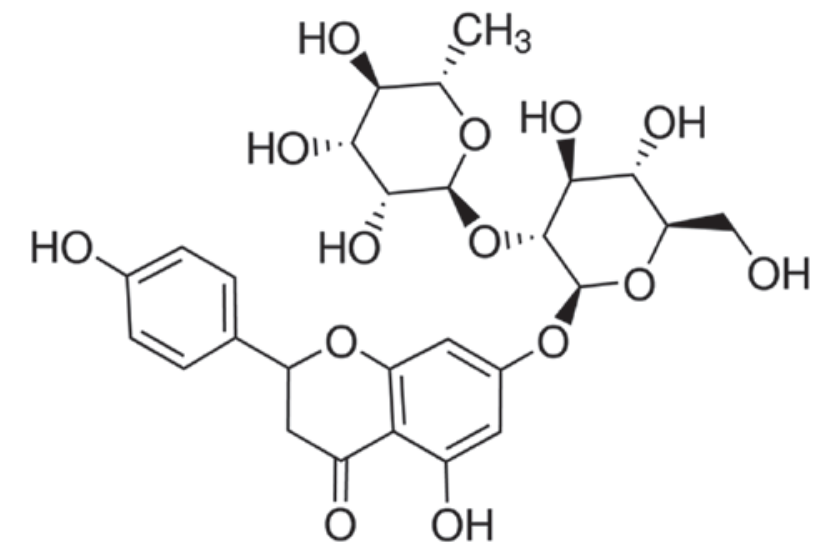

Figure 1. Chemical structure of naringin.

Western blot analysis of signal transducer and activator of transcription 3 (STAT3) and Janus kinase 2 (JAK2) protein expressions in AS mouse model. After the treatment with naringin for 3 days, $10 \mathrm{mg}$ AS tissue samples were removed, and incubated with $100 \mu \mathrm{l}$ tissue lysis buffer (Thomas Scientific, Swedesboro, NJ, USA) for $30 \mathrm{~min}$ on ice. The protein concentration was measured using a BCA kit (Bio-Rad Laboratories, Inc.). Equal quantities of protein $(50 \mu \mathrm{g})$ were resolved using $12 \%$ SDS-polyacrylamide gel and transferred onto a polyvinylidene fluoride membrane (Bio-Rad Laboratories, Inc). Next, the membrane was blocked with 5\% non-fat milk and incubated with anti-p-STAT3 (sc-135649; 1:2,000), anti-p-JAK2 (sc-16566-R; 1: 3,000) and $\beta$-actin (sc-130657; 1:5,000; all purchased from Santa Cruz Biotechnology, Inc., Dallas, TX, USA) and incubated overnight at $4^{\circ} \mathrm{C}$ followed by incubation with an anti-rabbit secondary antibody (sc-2795; 1:5,000, Santa Cruz Biotechnology Inc.) for $30 \mathrm{~min}$ at $37^{\circ} \mathrm{C}$. The signal was developed using an EasyBlot ECL kit (Shanghai Sangon Biological Engineering Technology \& Services Co., Ltd., Shanghai, China) according to the manufacturer's instructions. Densitometric measurement of the band intensity was performed with Quantity One software, version 4.4.0 (Bio-Rad Laboratories, Inc.).

Statistical analysis. Values were expressed as the mean \pm standard deviation and analysed using SPSS version 19.0, IBM SPSS, Inc., Chicago, IL, USA. One-way analysis of variance was performed for the statistical analysis of data using GraphPad Prism 5 software (GraphPad Software, San Diego, CA, USA). The Student's t-test was used to analyze statistical significance. $\mathrm{P}<0.05$ was considered to indicate a statistically significant difference.

\section{Results}

Effect of naringin on $O C, A L P$ and TG activity in AS mouse model. To determine the protective effect of naringin on ossification in the AS mouse model, the activity OC, ALP and TG were evaluated. OC and ALP activity were found to be decreased, while the TG activity was enhanced in the AS mouse compared with the control (Fig. 2). Pretreatment with naringin (20, 40 and $80 \mathrm{mg} / \mathrm{kg}$ ) markedly altered the OC and ALP activity, and reduced the TG activity in the AS mice (Fig. 2). 

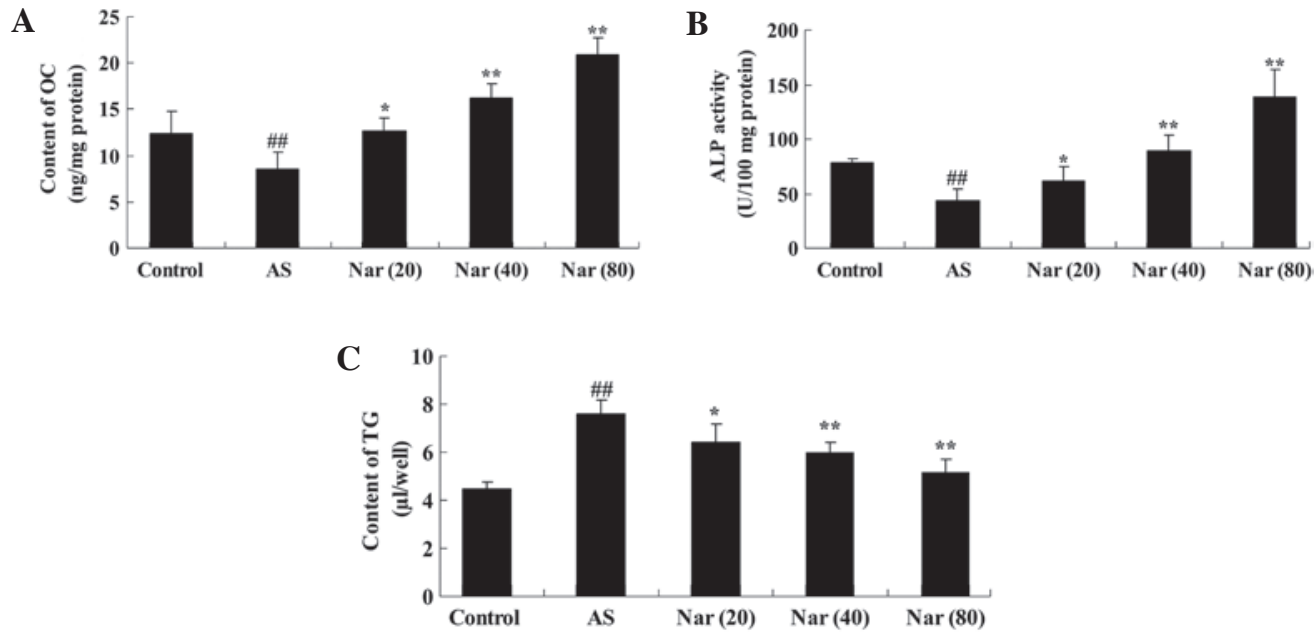

Figure 2. Effect of naringin on OC, ALP and TG activity values in AS mouse model. The protective effect of naringin on (A) OC, (B) ALP and (C) TG activity values in the AS mouse model. ${ }^{\# \#} \mathrm{P}<0.01$ vs. control group; ${ }^{*} \mathrm{P}<0.05$ vs. AS group; ${ }^{* *} \mathrm{P}<0.01$ vs. AS group. OC, osteocalcin; AS, ankylosing spondylitis group; Nar (20), naringin (20 mg/kg)-treated; Nar (40), naringin (40 mg/kg)-treated; Nar (80), naringin (80 mg/kg)-treated; One-way analysis of variance was performed for the statistical analysis of data, the Student's t-test was used to analyze statistical significance. ALP, alkaline phosphatase; TG, triglyceride.

A

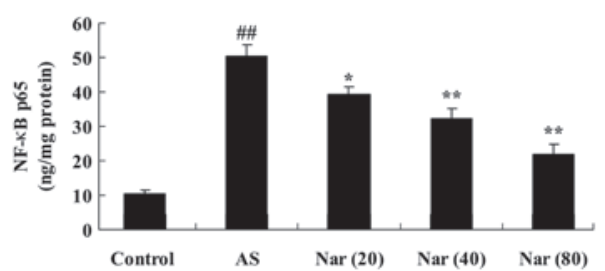

C

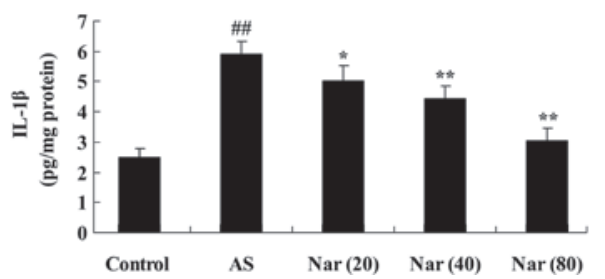

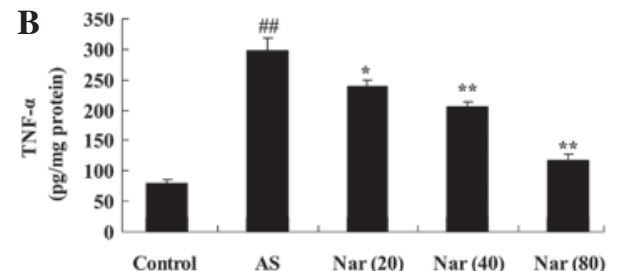

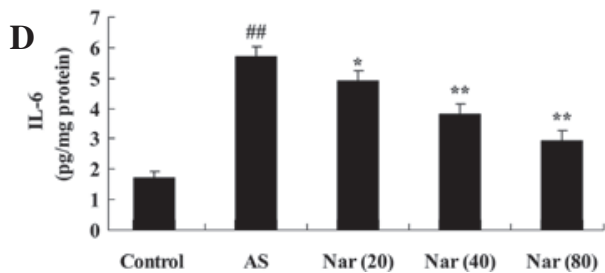

Figure 3. Effect of naringin on inflammatory factors in AS mouse model. The protective effect of naringin on the activity (A) NF- $\kappa \mathrm{B}$ p65 unit, (B) TNF- $\alpha$, (C) IL-1 $\beta$ and (D) IL-6 values in the AS mouse model. ${ }^{\# \#} \mathrm{P}<0.01$ vs. control group; ${ }^{*} \mathrm{P}<0.05$ vs. AS group; ${ }^{*} \mathrm{P}<0.01 \mathrm{vs}$. AS group. NF- $\kappa \mathrm{B}$ p65, nuclear factor- $\kappa \mathrm{B}$ p65; AS, ankylosing spondylitis group; Nar (20), naringin (20 mg/kg)-treated; Nar (40), naringin (40 mg/kg)-treated; Nar (80), naringin (80 mg/kg)-treated; One way analysis of variance was performed for the statistical analysis of data, the Student's t-test was used to analyze statistical significance. TNF- $\alpha$, tumor necrosis factor- $\alpha$; IL, interleukin.

A
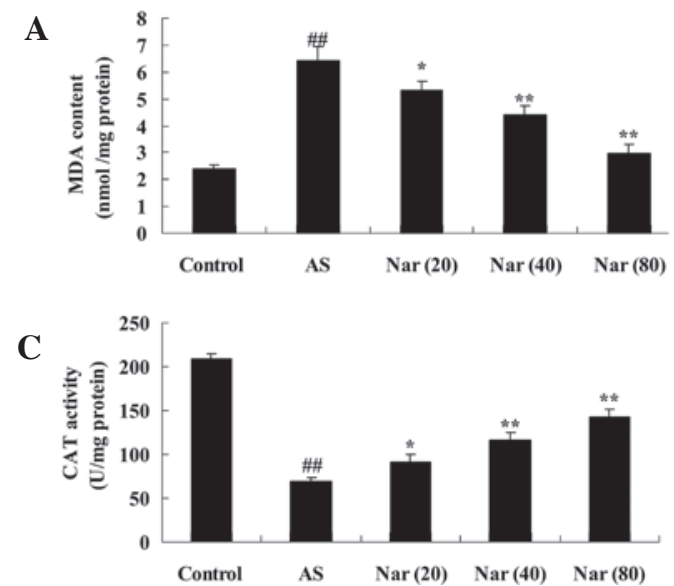
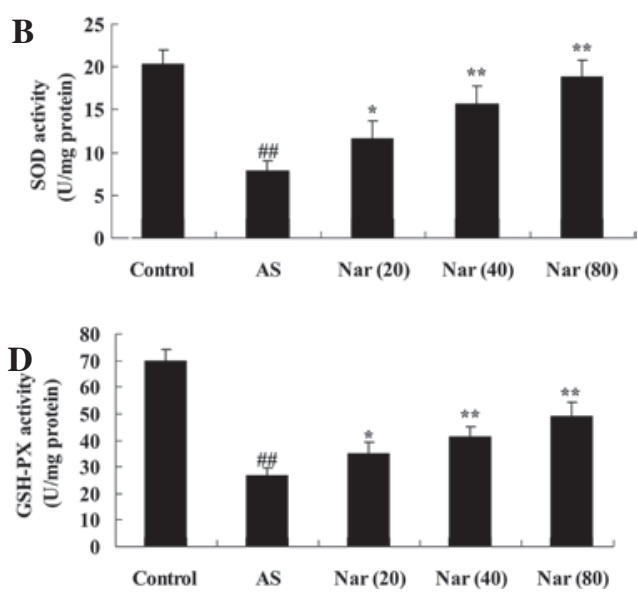

Figure 4. Effect of naringin on oxidative stress markers in AS mouse model. The protective effect of naringin on the concentrations of (A) MDA, (B) SOD, (C) CAT and (D) GSH-PX in the AS mouse model. ${ }^{\# *} \mathrm{P}<0.01$ vs. control group; ${ }^{*} \mathrm{P}<0.05$ vs. AS group; ${ }^{* *} \mathrm{P}<0.01$ vs. AS group. MDA, malondialdehyde; AS, ankylosing spondylitis group; Nar (20), naringin (20 mg/kg)-treated; Nar (40), naringin (40 mg/kg)-treated; Nar (80), naringin (80 mg/kg)-treated; One way analysis of variance was performed for the statistical analysis of data, the Student's t-test was used to analyze statistical significance. SOD, superoxide dismutase; CAT, catalase; GSH-PX, glutathione peroxidase. 
A

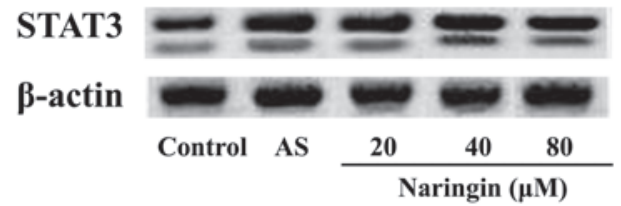

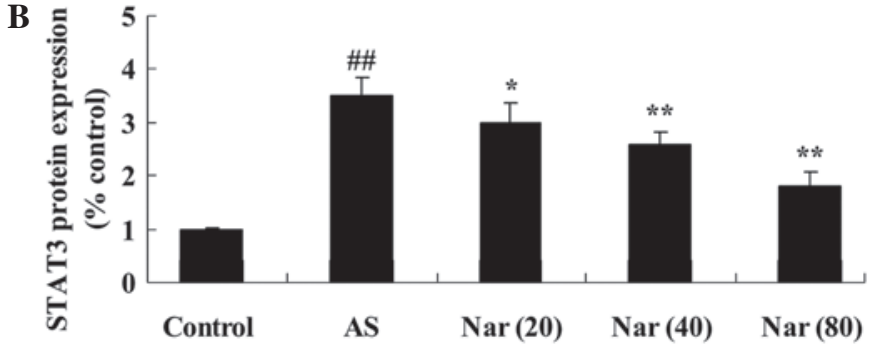

Figure 5. Effect of naringin on STAT3 protein expression in AS mouse model. (A) Western blot assay showing STAT3 protein expression and (B) statistical analysis of STAT3 protein expression in the AS mouse model. ${ }^{\# \#} \mathrm{P}<0.01$ vs. control group; ${ }^{*} \mathrm{P}<0.05$ vs. AS group; ${ }^{* *} \mathrm{P}<0.01$ vs. AS group. STAT3, signal transducer and activator of transcription 3; AS, ankylosing spondylitis group; Nar (20), naringin (20 mg/kg)-treated; Nar (40), naringin (40 mg/kg)-treated; $\operatorname{Nar}(80)$, naringin $(80 \mathrm{mg} / \mathrm{kg})$-treated. One-way analysis of variance was performed for the statistical analysis of data, the Student's t-test was used to analyze statistical significance.

A

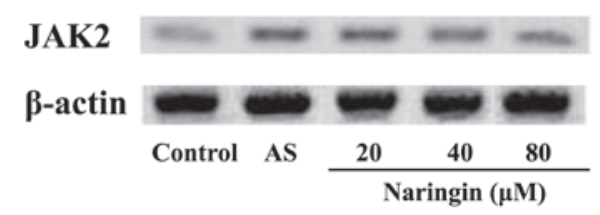

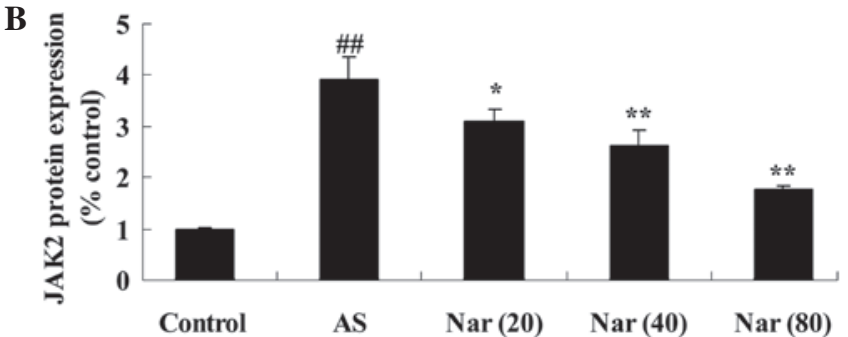

Figure 6. Effect of naringin on JAK2 protein expression in AS mouse model. (A) Western blot assay showing JAK2 protein expression and (B) statistical analysis of JAK2 protein expression in the AS mouse model. ${ }^{\# \#} \mathrm{P}<0.01$ vs. control group; ${ }^{*} \mathrm{P}<0.05$ vs. AS group; ${ }^{* *} \mathrm{P}<0.01$ vs. AS group. JAK2, Janus kinase 2 ; AS, ankylosing spondylitis group; Nar (20), naringin (20 mg/kg)-treated; Nar (40), naringin (40 mg/kg)-treated; Nar (80), naringin (80 mg/kg)-treated. One-way analysis of variance was performed for the statistical analysis of data, the Student's t-test was used to analyze statistical significance.

Effect of naringin on inflammatory factor expression in AS model mice. To determined the protective effect of naringin against the expression of inflammatory factors in the AS mouse model, the activity NF- $\mathrm{KB}$ p 65 unit, TNF- $\alpha$, IL- $1 \beta$ and IL-6 values were measured. As shown in Fig. 3, NF- $\kappa$ B p65 unit, TNF- $\alpha$, IL-1 $\beta$ and IL- 6 activity values were increased in the AS mouse group compared with the control. When pretreated with naringin $(20,40$ and $80 \mathrm{mg} / \mathrm{kg})$ for 8 days, NF- $\kappa$ B p 65 unit, TNF- $\alpha$, IL-1 $\beta$ and IL- 6 activity values were notably attenuated in the AS mice (Fig. 3).

Effect of naringin on markers of oxidative stress in AS mouse model. To infer the protective effect of naringin on oxidative stress of AS mouse model, the activity of MDA, SOD, CAT and GSH-PX were evaluated. In the AS group, the MDA activity was elevated, while the SOD, CAT and GSH-PX activities were reduced compared with the control (Fig. 4). The MDA, SOD, CAT and GSH-PX activities were improved by treatment with naringin (20, 40 and $80 \mathrm{mg} / \mathrm{kg}$ ) (Fig. 4).

Effect of naringin on STAT3 protein expression in AS mouse model. To elucidate the protective effect of naringin on STAT3, STAT3 protein expression of AS mouse was tested using a western blot assay. The STAT3 protein expression was induced in AS mouse (Fig. 5). However, treatment with naringin (20, 40 and $80 \mathrm{mg} / \mathrm{kg}$ ) mitigated this increased expression of STAT3 protein in AS mouse (Fig. 5).

Protective effect of naringin on JAK2 protein expression in AS mouse model. To clarify the involvement the protective effect of naringin on JAK2, JAK2 protein expression of AS mouse was evaluated. The JAK2 protein expression was induced in AS mice; however, treatment with naringin $(20,40$ and $80 \mathrm{mg} / \mathrm{kg}$ ) decreased this enhanced JAK2 expression (Fig. 6).

\section{Discussion}

AS is a chronic inflammatory disease, in which the rheumatoid factor is important, and it involves axial bone joints and tendon ligament attachment points, and the lesion is finally developed into the fibrosis of central joint and ankylosis, forming a typical 'bamboo vertebrae' (19). The pathogenesis of AS is currently unclear; however, its occurrence is associated with heredity, chronic infections, autoimmune disorders and endocrine disorders. The joint changes of AS primarily include synovial thickening and infiltration of macrophages, lymphocytes and plasma cells, accompanied by joint fibrosis and bone ankylosis (20). The primary site of the lesion is the region where the ligaments and joint capsule are attached, in which the inflammation leads to bone destruction, defects and replacement by connective tissue containing lymph and plasma cells; the filled and repaired cancellous bone develops ligament ossification in eroded bone surface (21).

The present study showed that administration of naringin increased OC and ALP activity, while reducing TG activity in AS model mice. These results are consistent with those of previous studies; for example, Liu et al suggested that the effects of naringin upregulates osteogenesis in human amniotic fluid-derived stem cells (22). Furthermore, $\mathrm{Li}$ et al reported that naringin is able to significantly improved ALP activity as well as upregulate the expression of type I collagen in the osteoblastic cell line MC3T3-E1 (23). 
AS is a type of rheumatism characterized by chronic inflammation of the axial joint, and may involve the internal organs and other tissues (24). Most scholars believe that this disease is an autoimmune inflammation reaction caused predominantly by genetic factors, in addition to being stimulated by trauma, infection, fatigue and other environmental factors $(25-27)$. The present results showed that naringin attenuates NF- $\kappa$ B p 65 unit, TNF- $\alpha$, IL-1 $\beta$ and IL- 6 activity in AS mice. These results are consistent with a previous study in which naringin was shown to ameliorate oxidative stress and inflammation in mice (28). In addition, it has been suggested that naringin may exert anti-inflammatory effects in the adult brain (29). However, the detailed mechanisms underlying the anti-inflammatory effects of naringin in AS mouse remain unclear, and further clarification is required in future.

In patients with AS, neutrophils are activated so that reactive oxygen species are generated, leading to oxidative stress (9). As a result of the increase in myeloperoxidase activity and advanced oxidation protein products in patients, the sulfhydryl level is decreased, from which it may be inferred that activated neutrophils serve a crucial function in the pathogenesis of AS (30). In the blood of patients with active AS, MDA levels and catalase activity are increased compared with those in control group (9). This catalase activity is positively correlated with erythrocyte sedimentation rate and C-reactive protein levels, and it is believed that the increase of catalase activity is a response to increased superoxide anion (31). The present results indicated that naringin reduced the MDA activity and increased the SOD, CAT and GSH-PX activities of the AS mice. Chen et al indicated that naringin had effective protection against paraquat-induced acute lung injury and pulmonary fibrosis through increasing activities of SOD, GSH-PX in mice (32). Cui et al suggested that naringin benefited the recovery of traumatic brain injury by reducing oxidative and inflammatory alterations in mice (33).

STATs are a group of cytoplasmic protein transcription factors, mediating the cytoplasm, which play a key role in the signaling of the nucleus (34). It has been reported that mutant mice lacking STAT3 are highly sensitive to AS, and the concentrations of serum inflammatory cytokines such as TNF- $\alpha$, IL-1 $\beta$ and IL-6 are increased (35). Furthermore, macrophages lacking in STAT3 show abnormal activation phenotypes, such as increased production of inflammatory cytokines in response to endotoxin (36). STAT3 activation is crucial for the prevention of chronic inflammation in mice (37). The present results showed that naringin inhibited the STAT3 protein expression in AS rat. A previous study showed that naringin inhibited the development of carrageenan-induced acute lung inflammation via suppression of STAT3 (38).

AK2 belongs to Janus kinase family, and the gene is located in the short arm of chromosome 9 (9p24), belonging to JAK family together with JAK1, JAK3 and TYK2 as intracellular protein tyrosine kinase (39). Under normal physiological conditions, JAK2 mediates the signal transduction of a variety of cytokines, including erythropoietin, thrombopoietin, granulocyte-macrophage colony stimulating factor and IL-3, thus regulating and promoting cell proliferation (40). The present study showed that naringin also inhibited JAK 2 protein expression in AS rat. In addition, naringin appeared to exert an anti-inflammatory effect via the suppression of the JAK2/STAT3 signaling pathway.

In summary, naringin exerted notable osteogenic, anti-inflammatory and anti-oxidative effects, and the mechanism was mediated by the downregulation of the JAK2/STAT3 signaling pathways in AS mice. Future studies are required to investigate the protective effect of naringin against AS.

\section{References}

1. Liu YF, Dong H, Tu SH, Zheng CH, Liu PL and $\mathrm{Hu}$ YH: Etanercept in the treatment of ankylosing spondylitis: A systematic review and meta-analysis. Exp Ther Med 8: 1585-1592, 2014

2. Chou CT, Huo AP, Chang HN, Tsai CY, Chen WS and Wang HP: Cytokine production from peripheral blood mononuclear cells in patients with ankylosing spondylitis and their first-degree relatives. Arch Med Res 38: 190-195, 2007.

3. Zhou L, Zhang Y, Xu H, et al: Decreased programmed death-1 expression on the $\mathrm{T}$ cells of patients with ankylosing spondylitis. Am J Med Sci 349: 488-492, 2015.

4. Rednic S, Marinescu C, Chira R, Rogojan L and Rednic N: Treatment with infliximab in a patient with ankylosing spondylitis and Crohn's disease. J Gastrointestin Liver Dis 15: 379-382, 2006.

5. Mielants H, Veys EM, Cuvelier C, et al: Gut inflammation in children with late onset pauciarticular juvenile chronic arthritis and evolution to adult spondyloarthropathy--a prospective study. J Rheumatol 20: 1567-1572, 1993.

6. Weber U, Hodler J, Kubik RA, Rufibach K, Lambert RG, Kissling RO, Pfirrmann CW and Maksymowych WP: Sensitivity and specificity of spinal inflammatory lesions assessed by whole-body magnetic resonance imaging in patients with ankylosing spondylitis or recent-onset inflammatory back pain. Arthritis Rheum 61: 900-908, 2009.

7. Maksymowych WP, Chiowchanwisawakit P, Clare T, Pedersen SJ, Østergaard M and Lambert RG: Inflammatory lesions of the spine on magnetic resonance imaging predict the development of new syndesmophytes in ankylosing spondylitis: Evidence of a relationship between inflammation and new bone formation. Arthritis Rheum 60: 93-102, 2009.

8. Okano K, Kimura K, Tanaka Y, Tsuchiya K, Akiba T and Nitta K: Direct measurement of reactive oxygen species in leukocytes during hemodialysis therapy. Int J Clin Exp Med 8: 20959-20964, 2015.

9. Kozaci LD, Sari I, Alacacioglu A, Akar S and Akkoc N: Evaluation of inflammation and oxidative stress in ankylosing spondylitis: A role for macrophage migration inhibitory factor. Mod Rheumatol 20: 34-39, 2010.

10. Jagetia GC, Reddy TK, Venkatesha VA and Kedlaya R: Influence of naringin on ferric iron induced oxidative damage in vitro. Clin Chim Acta 347: 189-197, 2004.

11. Zhou X, Zhang P, Zhang $\mathrm{C}$ and Zhu Z: Promotion of bone formation by naringin in a titanium particle-induced diabetic murine calvarial osteolysis model. J Orthop Res 28: 451-456, 2010.

12. Kim SY, Kim HJ, Lee MK, Jeon SM, Do GM, Kwon EY, Cho YY, Kim DJ, Jeong KS, Park YB, et al: Naringin time-dependently lowers hepatic cholesterol biosynthesis and plasma cholesterol in rats fed high-fat and high-cholesterol diet. J Med Food 9: 582-586, 2006.

13. Choe SC, Kim HS, Jeong TS, Bok SH and Park YB: Naringin has an antiatherogenic effect with the inhibition of intercellular adhesion molecule-1 in hypercholesterolemic rabbits. J Cardiovasc Pharmacol 38: 947-955, 2001.

14. Fernandez SP, Nguyen M, Yow TT, Chu C, Johnston GA, Hanrahan JR and Chebib M: The flavonoid glycosides, myricitrin, gossypin and naringin exert anxiolytic action in mice. Neurochem Res 34: 1867-1875, 2009.

15. Camargo CA, Gomes-Marcondes MC, Wutzki NC and Aoyama H: Naringin inhibits tumor growth and reduces interleukin-6 and tumor necrosis factor alpha levels in rats with Walker 256 carcinosarcoma. Anticancer Res 32: 129-133, 2012.

16. Liu Q, Lu L and Xiao M: Cell surface engineering of $\alpha-1-$ rhamnosidase for naringin hydrolysis. Bioresour Technol 123: 144-149, 2012. 
17. $\mathrm{Gu} \mathrm{X}, \mathrm{Wu} \mathrm{H}$ and $\mathrm{Fu} \mathrm{P}$ : Allicin attenuates inflammation and suppresses HLA-B27 protein expression in ankylosing spondylitis mice. Biomed Res Int 2013: 171573, 2013.

18. Kumar VS, Rajmane AR, Adil M, Kandhare AD, Ghosh P and Bodhankar SL: Naringin ameliorates acetic acid induced colitis through modulation of endogenous oxido-nitrosative balance and DNA damage in rats. J Biomed Res 28: 132-145, 2014.

19. Shiau MY, Lo MK, Chang CP, Yang TP, Ho KT and Chang YH: Association of tumour necrosis factor alpha promoter polymorphisms with ankylosing spondylitis in Taiwan. Ann Rheum Dis 66: 562-563, 2007.

20. Tam LS, Chan KY and Li EK: The influence of illness and variables associated with functional limitations in Chinese patients with ankylosing spondylitis. J Rheumatol 34: 1032-1039, 2007.

21. Gu X, Wu $\mathrm{H}$ and Fu P: Allicin attenuates inflammation and suppresses HLA-B27 protein expression in ankylosing spondylitis mice. Biomed Res Int 2013: 171573, 2013.

22. Liu M, Li Y and Yang ST: Effects of naringin on the proliferation and osteogenic differentiation of human amniotic fluid-derived stem cells. J Tissue Eng Regen Med, 2014 (Epub ahead of print).

23. Li L, Zeng Z and Cai G: Comparison of neoeriocitrin and naringin on proliferation and osteogenic differentiation in MC3T3-E1. Phytomedicine 18: 985-989, 2011.

24. Lange U, Teichmann J and Stracke H: Correlation between plasma TNF-alpha, IGF-1, biochemical markers of bone metabolism, markers of inflammation/disease activity and clinical manifestations in ankylosing spondylitis. Eur J Med Res 5: 507-511, 2000.

25. Schulz M, Dotzlaw H and Neeck G: Ankylosing spondylitis and rheumatoid arthritis: serum levels of TNF-alpha and Its soluble receptors during the course of therapy with etanercept and infliximab. Biomed Res Int 2014: 675108, 2014.

26. Huang WN, Tso TK, Kuo YC and Tsay GJ: Distinct impacts of syndesmophyte formation on male and female patients with ankylosing spondylitis. Int J Rheum Dis 15: 163-168, 2012.

27. Liu C, Hong T, Shao M, Chen Z and Wang C: Melatonin synergized with cyclosporine A improves cardiac allograft survival by suppressing inflammation and apoptosis. Mol Med Rep 10: 1323-1328, 2014.

28. Golechha M, Sarangal V, Bhatia J, Chaudhry U, Saluja D and Arya DS: Naringin ameliorates pentylenetetrazol-induced seizures and associated oxidative stress, inflammation, and cognitive impairment in rats: Possible mechanisms of neuroprotection. Epilepsy Behav 41: 98-102, 2014.
29. Jung UJ and Kim SR: Effects of naringin, a flavanone glycoside in grapefruits and citrus fruits, on the nigrostriatal dopaminergic projection in the adult brain. Neural Regen Res 9: 1514-1517, 2014.

30. Feijoo M, Tunez I, Tasset I, Montilla P, Ruiz A and Collantes E: Infliximab reduces oxidative stress in ankylosing spondylitis. Clin Exp Rheumatol 27: 167-168, 2009.

31. Karakoc M, Altindag O, Keles H, Soran N and Selek S: Serum oxidative-antioxidative status in patients with ankylosing spondilitis. Rheumatol Int 27: 1131-1134, 2007.

32. Chen Y, Nie YC, Luo YL, Lin F, Zheng YF, Cheng GH, Wu H, Zhang KJ, Su WW, Shen JG and Li PB: Protective effects of naringin against paraquat-induced acute lung injury and pulmonary fibrosis in mice. Food Chem Toxicol 58: 133-140, 2013.

33. Cui QJ, Wang LY, Wei ZX and Qu WS: Continual naringin treatment benefits the recovery of traumatic brain injury in rats through reducing oxidative and inflammatory alterations. Neurochem Res 39: 1254-1262, 2014.

34. Kicinska A, Leluk J and Jarmuszkiewicz W: Acanthamoeba castellanii STAT protein. PLoS One 9: e111345, 2014

35. Danoy P, Pryce K, Hadler J, Bradbury LA, Farrar C, Pointon J; Australo-Anglo-American Spondyloarthritis Consortium, Ward M, Weisman M, Reveille JD, et al: Association of variants at $1 \mathrm{q} 32$ and STAT3 with ankylosing spondylitis suggests genetic overlap with Crohn's disease. PLoS Genet 6: e1001195, 2010.

36. Ahmad SF, Attia SM, Bakheet SA, Zoheir KM, Ansari MA, Korashy HM, Abdel-Hamied HE, Ashour AE and Abd-Allah AR: Naringin attenuates the development of carrageenan-induced acute lung inflammation through inhibition of NF- $\mathrm{kb}$, STAT3 and pro-inflammatory mediators and enhancement of $\mathrm{I} \kappa \mathrm{B} \alpha$ and anti-inflammatory cytokines. Inflammation 38: 846-857, 2015.

37. Davidson SI, Liu Y, Danoy PA, Wu X, Thomas GP, Jiang L, Sun L, Wang N, Han J, Han H, et al: Association of STAT3 and TNFRSF1A with ankylosing spondylitis in Han Chinese. Ann Rheum Dis 70: 289-292, 2011.

38. Zimmers TA, Fishel ML and Bonetto A: STAT3 in the Systemic Inflammation of Cancer Cachexia. Semin Cell Dev Biol2016.

39. Chen C, Zhang X and Wang Y: Analysis of JAK 2 and STAT3 polymorphisms in patients with ankylosing spondylitis in Chinese Han population. Clin Immunol 136: 442-446, 2010.

40. Liu H, Yang Z, Hu J, et al: Improvement of thoracic aortic vasoreactivity by continuous and intermittent exercise in high-fat diet-induced obese rats. Biomed Rep 3: 527-532, 2015. 